\title{
DETERMINANTES DOS NOVOS CONTRATOS DE FOMENTO FLORESTAL NA MESORREGIÃO DO VALE DO RIO DOCE, MINAS GERAIS ${ }^{1}$
}

\author{
Uemerson Rodrigues de Souza ${ }^{2}$, Fabiano Luiz da Silva ${ }^{3}$, James Jackson Griffith ${ }^{4}$, João Eustáquio de Lima ${ }^{5}$, \\ Mirelle Cristina Abreu Quintela ${ }^{2}$ e Ederson Cláudio Vieira Costa ${ }^{2}$
}

\begin{abstract}
RESUMO - O setor florestal tem exercido importante papel no desenvolvimento socioeconômico das regiões produtoras de madeira. Os benefícios são atribuídos à geração de renda (alternativa para pequenos e médios produtores rurais), impostos, divisas e incremento no PIB. As novas exigências do mercado, em termos de competitividade, levaram as empresas a criarem os programas de fomento florestal como forma de expandir a oferta de madeira e garantir sua base produtiva. Nesse contexto, surge a necessidade de se identificarem os fatores que determinam a realização de novos contratos de fomento florestal. A amostra estudada foi composta pelos produtores rurais da mesorregião do Vale do Rio Doce, Minas Gerais, que realizaram pelo menos um contrato de fomento entre os anos de 1995 e 2006. Os dados foram coletados por meio de questionários semiestruturados no ano de 2006. Para identificar os fatores, utilizou-se a Análise Estatística Multivariada, especificamente a técnica de análise de discriminante. Os resultados apontaram que o sistema de medição da madeira (SISMED), a área contratada (ACONTRAT) e o tamanho da propriedade (TAMPROP) foram, nessa ordem, os principais determinantes para a realização de novos contratos. Desse modo, sugere-se que as empresas florestais desenvolvam programas de acompanhamento técnico e formação dos produtores fomentados, no intuito de reduzir as desconfianças no SISMED e aumentar o volume de áreas plantadas por meio do fomento.
\end{abstract}

Palavras-chave: Contratos, análise discriminante e fomento florestal.

\section{DETERMINING FACTORS FOR NEW PRIVATE WOODLOT CONTRACTS IN THE VALE DO RIO DOCE MESO REGIONIN MINAS GERAIS}

\begin{abstract}
The forestry sector has played an important role in the socio-economic development of woodproducing regions. Benefits include private income (an alternative for small and medium-sized rural producers), government tax income, balance of payment advantages, and increasing GNP. New market demands in terms of competitiveness have led cellulose companies to create private woodlot programs as a way to expand timber supplies and guarantee a productive basis. Within this context, arises the need to identify determinant factors for signing new private woodlot contracts. The sample studied is composed of rural producers who signed at least one contract in the Vale do Rio Doce meso region in Minas Gerais in 1995-2006.Data were collected in 2006 using a semi-structured questionnaire. Factors were identified using a multivariate statistical analysis, specifically applying a discriminate analysis technique. Results indicate that the system used by the company to measure timber (SISMED), contracted area size (ACONTRAT) and property size (TAMPROP) are, in this order, the principal determinants for assuming new contracts. Based on the study results, it is suggested that forestry companies further develop technical monitoring and training for woodlot producers. These programs could reduce the present lack of confidence indicated for the SISMED factor and help increase the amount of areas planted.
\end{abstract}

Keywords: Contracts, discriminant analysis and forest promotion.

\footnotetext{
${ }^{1}$ Recebido em 29.08.2007 e aceito para publicação em 06.03.2009.

${ }^{2}$ Programa de Pós-Graduação em Economia Rural da Universidade Federal de Viçosa (UFV). E-mail: <uemersoncoop@ yahoo.com.br>.

${ }^{3}$ Programa de Pós-Graduação em Ciência Florestal da UFV. E-mail: <fabianocoop@ yahoo.com.br>.

${ }^{4}$ Departamento de Engenharia Florestal da UFV. E-mail: <griffith@ufv.br>.

${ }^{5}$ Departamento de Economia Rural da UFV. E-mail: <jelima@ufv.br>.
} 


\section{INTRODUÇÃO}

O setor florestal brasileiro tem exercido importante papel para o desenvolvimento socioeconômico do país. Os benefícios do setor são atribuídos à geração de emprego (que ocorrem tanto no meio rural quanto no urbano), arrecadação de impostos, geração de divisas e crescimento do produto interno bruto - PIB (VALVERDE, 2003).

Segundo a Sociedade Brasileira de Silvicultura - SBS (2006), o setor contribuiu com 5\% do Produto Interno Bruto - PIB, gerando US\$ 3 bi em impostos, US \$ 16 bi em exportações e mais de dois milhões de empregos. Em termos de ocupação no Brasil, a área de florestas plantadas compreende cerca de 5,6 milhões de hectares, e $72 \%$ desse total está concentrado nas Regiões Sul e Sudeste.

Além dos benefícios advindos do campo econômico, o setor florestal também contribui para recuperação de áreas desmatadas, viabiliza projetos de educação ambiental, proporciona melhorias na qualidade vida da população e constitui atividade econômica alternativa para os produtores rurais em diversas regiões.

Silva (1994) afirmou, porém, que o crescimento da atividade florestal gerou externalidades negativas no que se refere à configuração do espaço no meio rural. De acordo com esse autor, o fato de as empresas florestais investirem maciçamente na produção de madeira (motivadas pelos incentivos fiscais) ${ }^{1}$, criaram-se diversos latifúndios de monoculturas, e os produtores que venderam suas propriedades acabaram saindo do campo e transferindo-se para as cidades. Entretanto, na visão de Oliveira et al. (2006) os impactos negativos da concentração de terras poderiam ser minimizados em face de uma política de fomento florestal para os produtores rurais. Desse modo, além de manter a estrutura agrária no meio rural, a atividade florestal poderia ser empreendida, no intuito de gerar mais renda e aumentar a riqueza regional.

Nesse sentido, o fomento surge como importante instrumento de apoio à atividade florestal, tanto para as empresas fomentadoras quanto para os produtores rurais, que identificam nas atividades novas oportunidades de expansão de suas atividades.

\section{O FOMENTO FLORESTAL NO BRASIL}

Segundo Silva (2007) e Oliveira (2003), a atividade de fomento florestal consiste num sistema de fornecimento de matéria-prima pelas empresas de celulose e siderúrgicas, com os objetivos de estimular a produção e garantir a oferta de madeira. De acordo com os citados autores, nos contratos de fomento florestal, especificamente realizado pelas empresas de celulose e papel, são repassados aos produtores mudas clonais, fertilizantes, defensivos, recursos financeiros e assistência técnica; em contrapartida, os produtores são obrigados a comercializar $97 \%$ de sua produção com a empresa fomentadora. Caso os produtores desejem comercializar a madeira com outras empresas ou para outro segmento do setor, eles deverão arcar com os custos referentes à quebra do contrato de fomento florestal.

Silva (2007) aponta, assim, a existência de duas modalidades de contratos de fomento florestal ${ }^{2}$. A primeira é chamada de contrato de fomento comercial, que se caracteriza pelo fornecimento dos insumos e mão-de-obra por parte da empresa fomentadora. $\mathrm{O}$ monitoramento do plantio é realizado pela empresa fomentadora até o fim do segundo ano da floresta. A partir desse ponto, a condução da atividade (combate a pragas e proteção) é de responsabilidade do fomentado. Esses custos com mão-de-obra e monitoramento são calculados em volume de madeira e descontados na ocasião da comercialização com a indústria fomentadora.

A segunda modalidade é o contrato de fomento convencional. Nesse tipo de contrato, a empresa fomentadora fornece os insumos necessários para o plantio (adubos, formicidas e mudas clonais), e a contratação da mão-de-obra fica a cargo do fomentado. Nesse modelo de contrato, a empresa fomentadora antecipa ao produtor uma quantia monetária referente à área a ser plantada para custear o plantio, sendo esse valor descontado na ocasião da entrega da madeira. A exigência dos dois tipos de contratos é que o produtor

\footnotetext{
${ }^{1}$ Esses incentivos foram fornecidos pelo governo brasileiro até a década de 1980. Após a década de 1990, o setor passou a atuar em uma nova dinâmica, enfrentando os fatores competitivos do mercado da madeira.

${ }^{2}$ Os contratos de fomento florestal são realizados de acordo com as áreas a serem plantadas. Portanto, o mesmo produtor pode fazer mais de um contrato de fomento, ou seja, para áreas plantadas de diferentes tamanhos.
} 
se comprometa a entregar $97 \%$ da produção para a empresa, e, dessa forma, no contrato convencional os custos referentes aos insumos são bonificados.

Para os produtores rurais, o fomento viabiliza o início de uma nova atividade econômica sem a necessidade de um desembolso de capital inicial, que é benéfico devido à limitação de capital por parte dos produtores. Pelo lado das empresas, o fomento constitui-se numa forma de se integrar verticalmente sem realizar elevados investimentos na imobilização de terras e a custos menores $^{3}$ (SILVA, 2007).

Os rumos dos programas de fomento florestal no Brasil apresentam expectativas de crescimento nos próximos anos. Segundo a Associação de Produtores de Florestas Plantadas - ABRAF até 2005, as empresas associadas à ABRAF haviam desenvolvido o fomento florestal em mais de 400 municípios de diversos estados. Segundo a SBS (2006), a expectativa para os próximos anos é de aumento no número de contratos de fomento, especificamente por parte das empresas que atuam no setor de celulose e papel.

Nesse sentido, o objetivo deste estudo é identificar os fatores que determinam a realização de novos contratos de fomento florestal na mesorregião do Vale do Rio Doce em Minas Gerais, nos períodos de 1995 a 2006.

\section{MATERIAL E MÉTODOS}

Conforme exposto, este estudo visa identificar os fatores que influenciam a decisão dos produtores rurais em realizar novos contratos de fomento florestal. Com esse propósito foram formados dois grupos, sendo o primeiro composto por produtores que realizaram mais de um contrato e o segundo, por aqueles que fizeram apenas um contrato de fomento florestal.

Para se estabelecerem as diferenças entre os dois grupos previamente definidos, foi selecionada a técnica estatística de análise discriminante. Essa técnica tem sido utilizada em diversas áreas do conhecimento. Como exemplo, cita-se o trabalho na área de finanças de Gimenes e UripeOpazo (2002), que realizaram a previsão de solvência de cooperativas agropecuárias no Estado do Paraná.
No campo das ciências agrárias, Nanni et al. (2004) analisaram as diferenças no solo por meio de resposta espectral no nível terrestre. A análise discriminante foi utilizada por Gonçalves et al. (2008) para identificar elementos estratégicos que condicionam o desempenho da indústria brasileira e seu impacto econômico. E na área de comércio internacional Coelho et al. (2008) mensuraram o impacto do investimento direto estrangeiro (IDE) nas exportações do agronegócio brasileiro.

A aplicabilidade dessa técnica reforça sua importância e robustez para o estudo proposto, e por essa razão será apresentada mais pormenorizadamente no tópico seguinte.

\subsection{Análise Discriminante}

Esta análise é um ramo da estatística multivariada ${ }^{4}$ utilizada para realizar inferências sobre determinada população dividida em grupos. Segundo Mingote (2005), essa técnica consiste em diferenciar populações e, ou, classificar os objetos em populações prédefinidas.

A análise discriminante possui quatro funções principais: i) determinar uma ou mais funções que sejam capazes de efetuar a discriminação máxima entre duas populações; ii) testar a classificação prévia de um elemento (objeto) em determinada população; iii) estabelecer uma regra para alocar um ou mais elementos em populações distintas; e iv) identificar, no conjunto de variáveis observadas, aquelas que tem maior poder de discriminação entre os grupos.

Para tanto, Hair et al. (2005, p. 209) afirmaram que “[...] a discriminação é conseguida estabelecendo-se pesos das variáveis estatísticas para cada variável, para maximizar a variância entre os grupos relativa à variância dentro dos grupos, e a função discriminante é determinada por meio de uma equação". Essa equação consiste numa combinação linear das variáveis $X$ que representam os elementos de ambos os grupos e determinando os coeficientes $a_{1}, a_{2}, \ldots a_{p}$, que tornam máxima a homogeneidade dentro dos grupos e a heterogeneidade entre os grupos. Portanto, o objetivo é determinar os coeficientes que apresentam o poder de discriminação máximo da função. De acordo com

\footnotetext{
${ }^{3}$ A redução de custos é atribuída à mão-de-obra que é custeada pelo fomentado e à menor carga tributária incidente sobre a empresa.

${ }^{4}$ Outras técnicas de análise multivariada podem ser utilizadas para ordenar indivíduos em grupos. Entre elas, citam-se correlações canônicas, componentes principais, análise fatorial e análise de Cluster.
} 
Barroso e Artes (2003), o método mais utilizado para determinar os coeficientes é o método de Fischer, ou função discriminante de Fischer.

Para a determinação da função discriminante de Fischer é necessário cumprir quatro etapas principais. Inicialmente, definem-se $\tau_{1}$ e $\tau_{2}$ como os grupos que se deseja discriminar, sendo $n_{1}$ e $n_{2}$, respectivamente, o número de elementos pertencentes aos grupos $t_{1}$ e $t_{2}$. Define-se também um vetor de $p$ variáveis que representa os elementos dos grupos, chamado de $X^{\prime}=\left[\mathrm{x}_{1} \mathrm{x}_{2} \mathrm{x}_{3} \ldots \mathrm{x}_{\mathrm{p}}\right]$.

A segunda etapa trata-se da definição das matrizes de variância e covariância das variáveis $X$ das populações $\tau_{1}$ e $\tau_{2}$, ressaltando-se que as matrizes devem ser homogêneas em ambas as populações. Para obter a variância conjunta das populações, define-se uma matriz $S_{p}$ que representa a variância das populações conjuntamente como em $S_{1}$ e $S_{2}$. A matriz $S_{p}$ é obtida a partir de:

$$
S_{p}=\frac{\left(n_{1}-1\right) S_{1}+\left(n_{2}-1\right) S_{2}}{n_{1}+n_{2}-2}
$$

Nesse sentido, a função discriminante de Fischer consiste em determinar combinações lineares das variáveis $\left[\mathrm{x}_{1} \mathrm{x}_{2} \mathrm{x}_{3} \ldots \mathrm{x}_{\mathrm{p}}\right]$, as quais podem ser apresentadas por:

$$
Y=a^{\prime} x
$$

Dessa forma, os valores de $Y$ obtidos para cada elemento de $\tau_{1}$ são o máximo possível separados dos valores de $Y$ obtidos em $\tau_{2}$.

Conforme citado, a análise discriminante busca maximizar a homogeneidade dentro dos grupos e a heterogeneidade entre grupos. Portanto, para separar os grupos deve maximizar a diferença dos valores médios (ou centróides) de $Y$ calculados para os elementos das duas populações, dividido pelo desvio-padrão de $Y$. Essa equação é representada por:

$$
\frac{\overline{Y_{1}}-\overline{Y_{2}}}{S_{y}}
$$

Elevando (3) ao quadrado, tem-se:

$$
\frac{\left(\overline{Y_{1}}-\overline{Y_{2}}\right)^{2}}{s_{y}^{2}}=\text { Distância ao quadrado entre as médias/ }
$$

variância de $Y$
Considerando que $\overline{Y_{1}}=a^{\prime} \overline{X_{1}}$ e $\overline{Y_{2}}=a^{\prime} \overline{X_{2}}$, sendo $\overline{X_{1}}$ e $\overline{X_{2}}$ os vetores de médias das variáveis explicativas em $\tau_{1}$ e $\tau_{2}$, tem-se:

$$
\frac{\left(\overline{Y_{1}}-\overline{Y_{2}}\right)}{s_{y}^{2}}=\frac{\left(a^{\prime} \overline{X_{1}}-a^{\prime} \overline{X_{2}}\right)^{2}}{a^{\prime} S_{p} a}
$$

Portanto, a combinação linear que maximiza a razão expressa em (5) é dada por:

$$
Y=\left(\overline{X_{1}}-\overline{X_{2}}\right)^{T} S_{p}^{-1}
$$

A expressão (6) é notação matricial da função discriminante de Fisher para dois grupos. Igualando (6) a (2), tem-se que:

$$
a^{\prime}=\left(\overline{X_{1}}-\overline{X_{2}}\right)^{T} S_{p}^{-1}
$$

Algebricamente, a função discriminante linear tem a seguinte forma:

$Y=\beta_{0}+\beta_{1} X_{1}+\beta_{2} X_{2}+\beta_{i} X_{i}+\ldots . . \beta_{p-1} X_{p-1}=\beta_{0} \sum_{i=1}^{p-1} \beta_{i} X_{i}$

em que $Y$ é o escore (coeficiente) discriminante que funciona como modo de classificação, e $\beta_{l}, i=0,1 \ldots . p$ são os coeficientes da função discriminante nas variáveis $X_{1}, i=0,1 \ldots p, \operatorname{com} X_{0}=1$.

Para determinação do número máximo de funções (combinações lineares), observa-se o número mínimo de $p$ variáveis e $g-1$ grupos ou populações a serem discriminadas. Desse modo, quando se tratar de duas populações, tem-se apenas uma função.

Após a realização das classificações dos elementos, recomenda-se a realização do teste de adequabilidade dos elementos do modelo. O teste pode ser realizado a partir da Regra de Bayes, a qual leva em consideração a probabilidade de o indivíduo ser classificado em um grupo erroneamente, ou seja, o elemento ser classificado no grupo $\tau_{1}$, e na verdade ele pertencer ao grupo $\tau_{2}$ ou vice-versa.

A Figura 1 apresenta a forma como ocorre a classificação dos elementos nos dois grupos propostos. O escore de corte é representado pela letra $Z_{c}$, ou seja, este é o valor do coeficiente que separa as duas populações. No caso de o indivíduo pertencer ao grupo $\tau_{1}$ se seu escore $Y_{i} \leq Z_{c}$ e classificado no grupo $\tau_{2}$ se $Y \geq Z_{c}$. Dessa forma, se o escore do indivíduo $i$ for $Y_{i} \leq Z_{c}$ e ele pertencer ao grupo $\tau_{1}$, a probabilidade de uma 
classificação errônea é $\mathrm{P}(1 / 2)$ e dada pela área " $\mathrm{A}$ ". No caso contrário, se $Y_{i}>Z_{c}$ pertencer à população $\tau_{1}$, a probabilidade de classificação errada é de $\mathrm{P}(2 / 1)$ e representada pela área " $\mathrm{B}$ ".

\subsection{Operacionalização das variáveis}

Para realização deste estudo foi utilizada uma amostra dos produtores rurais da mesorregião do Vale do Rio Doce, Minas Gerais. A mesorregião é formada por sete microrregiões, sendo definidas cinco microrregiões como foco do programa de fomento desenvolvido pela empresa florestal. Os municípios polos regionais são: Ganhães, Ipatinga, Caratinga, Governador Valadares e Peçanha. O total de produtores fomentados até 2006 era de 733 produtores, distribuídos no total de 68 municípios da mesorregião. Considerando que houve um grupo de produtores que perderam o plantio (fatores climáticos) e outros que descumpriram o contrato, consideraram-se como população total 718 produtores.

A amostra foi definida a partir da sugestão de Gil (1999), representada pela formula (9).

$$
n=\frac{\sigma^{2} \cdot p \cdot q \cdot N}{e^{2}(N-1)+\sigma^{2} p \cdot q}
$$

Sendo:

$\sigma=2 ; \mathrm{p}=50 ; \mathrm{q}=50 ; \mathrm{N}=718 ; \mathrm{e}=10 \%$

$\mathrm{n}=$ tamanho da amostra;

$\sigma=$ nível de confiança escolhido, expresso em números de desvio-padrão;

$\mathrm{p}=$ porcentagem com a qual o fenômeno se verifica;

$\mathrm{q}=$ porcentagem complementar;

$\mathrm{N}=$ tamanho da população; e

e $=$ erro máximo permitido.

Dessa forma, o número total de produtores exigidos pelo método estatístico foi de 88 produtores rurais. Porém, para ampliar a segurança da amostra foram aplicados 141 questionários.

Os produtores rurais foram classificados em dois grupos a partir da relação contratual com a empresa. Portanto, o grupo 1 era composto por 90 produtores que realizaram mais de um contrato. $\mathrm{O}$ grupo 2 possuía 51 produtores, os quais realizaram apenas um contrato.

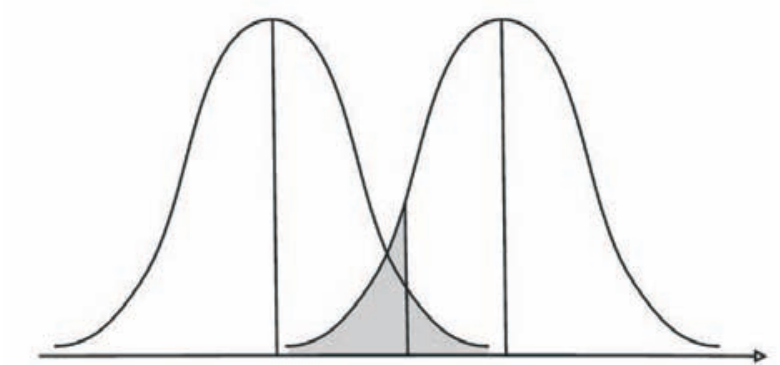

Fonte: PINARE, 1985.

Figura 1 - Representação de probabilidade de classificação errônea.

Figure 1 -Representation of error classification probability.

A definição das variáveis foi realizada no sentido de identificar os fatores que motivam os produtores a realizarem mais contratos e aqueles que influenciam contrariamente. As variáveis selecionadas foram:

CONT- $N^{\circ}$. de contratos realizados.

- TAMPROP- Tamanho da propriedade.

- ACONTRAT- Área contrata: Área que realmente foi plantada.

- SISMED- Sistema de medição da madeira.

IDADE- Idade do produtor.

- LOCAREGIO- Localização do produtor em relação à fábrica.

As varáveis citadas são organizadas da forma apresentada abaixo, e o objetivo da função discriminante é determinar os coeficientes betas.

$$
\begin{aligned}
& \text { CONT }(Y)=\beta_{1} \text { TAMPROP }+\beta_{2} \text { ACONTRAT }+ \\
& +\beta_{3} \text { SISMED }+\beta_{4} \text { IDADE }+\beta_{5} \text { LOCAREGIO }
\end{aligned}
$$

A variável dependente, ou de controle, foi definida como o número de contratos realizados pelos produtores. Dessa forma, para os produtores que realizaram mais de um contrato foi classificado como (1), caso tenha feito apenas um contrato (2). As variáveis independentes seguem nos parágrafos subsequentes.

As variáveis TAMPROP e ACONTRAT foram selecionadas com o objetivo de verificar se as características referentes à propriedade determinam a realização de novos contratos, ou seja, analisar se os produtores rurais com maior extensão de terras são mais propensos a realizarem novos contratos do que aqueles que possuem propriedades de menor tamanho.

R. Árvore, Viçosa-MG, v.33, n.2, p.377-386, 2009 
A área contratada (destinada ao fomento) foi selecionada no intuito de analisar a relação entre tamanho das áreas plantadas e novos contratos.

Outra variável selecionada refere-se ao sistema de medição adotado pela empresa fomentadora. O objetivo do uso dessa variável é identificar a receptividade do produtor em relação aos métodos de medição da madeira utilizado pela empresa.

Nessa análise, a idade do produtor também foi considerada como relevante para a determinação de novos contratos. E, por último, foi definida a variável que reflete a visão do produtor em relação à sua localização geográfica até a fábrica de celulose e papel. O objetivo de analisar essa variável é analisar a opinião dos produtores no que se refere à capacidade de a empresa pagar preços diferenciados, e, portanto, influenciar a decisão de realização de novos contratos.

\subsection{Fontes de Dados}

Os dados referentes a cada produtor foram levantados por meio de questionários, aplicados em 2006 . Os produtores foram escolhidos de forma aleatória, por meio de sorteio, com intuito de não direcionar a análise para algumas partes da mesorregião. Os dados foram processados utilizandose o software SPSS, versão 11.5.

\section{RESULTADOS E DISCUSSÕES}

A partir dos resultados da análise discriminante, verificou-se que 121 observações foram consideradas válidas pelo modelo, sendo 80 pertencentes ao grupo 1 e 41 pertencentes ao grupo 2. Depois foram realizados alguns testes para verificação da qualidade do ajustamento do modelo. O primeiro teste, recomendado por Marroco (2003, p. 351), foi o teste de igualdade das médias. O objetivo desse teste foi verificar se as médias das variáveis são estatisticamente iguais em cada grupo de produtores. Se caso as variáveis apresentarem médias iguais, isso significa que elas não explicam a diferença entre os grupos, ou seja, não contribuem para a discriminação dos objetos.

O resultado do teste é apresentado na Tabela 1, em que se identifica que as variáveis IDADE e LOCAREGIO apresentaram p-value (sig) maior que o á (nível de significância) de 0,05. Dessa forma, não se rejeita a hipótese nula de igualdade entre as médias dessas variáveis. Isso significa que a idade dos produtores e sua localização em relação à fábrica não contribuem para discriminar os grupos.

No que se refere à idade, pode-se inferir que os produtores já habituados com os tipos de contratos de fomento continuarão realizando novos contratos devido a outros fatores. Silva (2007) afirmou que, na visão dos produtores, esses fatores são os insumos e financiamentos oferecidos, retorno financeiro, sustentabilidade do agronegócio florestal, assistência técnica e baixo custo de mão-de-obra na atividade.

Em relação à localização do produtor, essa também não apresentou significância na análise. Entende-se que, devido ao fato de os produtores receberem preços diferenciados ${ }^{5}$ pela produção, ou seja, aqueles mais próximos à fábrica de celulose, eles possuem menos opções de venda da madeira e menor custo de transporte, portanto acabam recebendo menores preços pela madeira. Já os produtores localizados a distâncias maiores possuem maiores custos de transporte e outras oportunidades de comercialização da madeira (produção de carvão vegetal para a siderurgia e indústria moveleira, entre outras), o que leva a empresa fomentadora a pagar preços maiores pela produção.

Tabela 1 - Teste de igualdade das médias das variáveis dos grupos que realizaram um contrato e aqueles que fizeram mais de um

Table 1 - Equality test for variable averages of the groups that signed a contract and those that signed more than one

\begin{tabular}{lccc}
\hline Variáveis & Wilks' Lambda & Estatística F & Nível de Significância \\
\hline TAMPROP - Tamanho da propriedade & 0,961 & 4,803 & 0,03 \\
ACONTRAT - Área contratada & 0,92 & 10,411 & 0,002 \\
SISMED - Sistema de medição & 0,919 & 10,491 & 0,002 \\
IDADE - Idade do produtor & 0,983 & 2,081 & 0,152 \\
LOCAREGIO - Localização do produtor & 0,99 & 1,199 & 0,276 \\
\hline
\end{tabular}

Fonte: Dados da pesquisa.

${ }^{5} \mathrm{O}$ reduzido número de empresas que atuam no segmento de produção de celulose forma uma estrutura de mercado oligopolista, podendo influenciar os preços dos produtos florestais. Desse modo, as empresas caracterizam-se pelo exercício do poder de monopsônio, ou seja, influenciam os preços de compra da madeira.

R. Árvore, Viçosa-MG, v.33, n.2, p.377-386, 2009 
As condições expostas reafirmaram a importância do fomento para os produtores que estão localizados mais próximos das fábricas. Oliveira et al. (2006), analisando as práticas de fomento florestal no Estado do Espírito Santo, verificaram que a silvicultura constituise importante atividade econômica; além disso, os investimentos advindos do fomento contribuíram para a expansão de atividades econômicas secundárias, propiciando, por um lado, maior desenvolvimento local e, por outro, maior dependência dos recursos financiadores por parte dos produtores rurais.

A localização do produtor em relação à fábrica não foi considerada, desse modo, pelo modelo como fator relevante para discriminar os produtores que optaram pela realização de mais de um contrato de fomento florestal, fazendo-se necessária a análise de outros elementos que podem influenciar o comportamento dos produtores.

As variáveis que apresentaram significância estatística foram, portanto, TAMPROP, ACONTRAT e SISMED. Para a verificação da homogeneidade das matrizes de covariâncias das duas populações, foi realizado o teste $M$ de Box.

Após as considerações realizadas, estimou-se a função discriminante linear de Fischer para o grupo de produtores que realizaram novos contratos de fomento e aqueles que fizeram apenas um contrato. Na Tabela 2 , apresenta-se a função discriminante, que foi significativa no nível de $1 \%$ de significância. Foram utilizadas na análise variáveis padronizadas, que permitem identificar aquelas que possuem maior poder de discriminação dos objetos entre grupos. Nesse caso, as variáveis SISMED e a ACONTRAT apresentaramse como as que mais influenciam a decisão do produtor em realizar novos contratos.

A variável SISMED apresentou o sinal negativo (conforme esperado), mostrando que os produtores são avessos ao sistema de medida utilizado pela empresa fomentadora. Segundo Silva (2007), os produtores consideram o atual sistema (no pátio, medida em metros cúbicos e posto na fábrica peso/volume), de difícil entendimento e pouco confiável. Desse modo, o fato de os produtores não concordarem com sistema de medida diminui a probabilidade de realização de novos contratos.
Tabela 2 - Função discriminante dos grupos de produtores Table 2 -Discriminating function for the producer groups

\begin{tabular}{lccc}
\hline Função & SISMED & ACONTRAT & TAMPROP \\
\hline Discriminante (Y) & $-0,665$ & 0,602 & 0,135 \\
Teste de & $\lambda$ de Wilks & Estatística & $p$-value \\
significância & 0,84 & 20,254 & 0,001 \\
\hline
\end{tabular}

Fonte: Dados da pesquisa.

O fato de a variável SISMED apresentar o maior poder de discriminação $(0,665)$ revela a importância de se elaborarem políticas de acompanhamento técnico nas etapas de comercialização, de modo que os produtores possam ter garantia das quantidades que estão sendo transacionadas. Desse modo, verifica-se que, quanto maior o nível de satisfação dos produtores com os sistemas utilizados na medição, maiores as chances da realização de um novo contrato.

Silva (2007), analisando o fomento florestal em Minas Gerais, identificou que o desconhecimento sobre os métodos utilizados na medição da madeira influencia negativamente a confiança entre fomentados e empresa fomentadora, fazendo com esses não tenham motivação para realizar novos contratos.

Canto et al. (2006), analisando o processo de produção de madeira com produtores fomentados no Estado do Espírito Santo, detectaram que nas cinco regiões pesquisadas houve diferenças nos métodos de colheita, reforçando a hipótese de maior desconfiança por parte dos fomentados e também menores possibilidades de esses realizarem novos contratos de fomento.

Outro fator relevante apresentado pela função discriminante está relacionado com a área contratada ${ }^{6}$. De acordo com o coeficiente $(0,602)$, quanto maior a área contratada, maiores as chances de o produtor optar pela realização de novos contratos.

Esse resultado corrobora os de Silva (2007), demonstrando que os produtores visualizam retornos financeiros como elemento mais importante para aderir ao fomento. Em segundo lugar, o fato de o fomento viabilizar insumos e recursos para os investimentos iniciais contribui para o aumento das áreas contratadas, considerando-se que os produtores possuem baixo

${ }^{6}$ A área contratada refere-se à área efetivamente plantada pelo produtor. Quando o produtor opta pelo fomento, ele disponibiliza determinado tamanho para o plantio. A área contratada é o tamanho real plantado, de acordo com as condições técnicas. 
capital para investirem na expansão da produção de madeira. Desse modo, quanto maior a área contratada, maiores as chances de realização de novos contratos.

Juntamente com a variável ACONTRA, a função apresenta a TAMPROP como fator relevante da análise. O coeficiente estimado $(0,135)$ apresenta sinal coerente com o esperado de que o tamanho da propriedade possui relação positiva com as chances de realização de novos contratos. Desse modo, entende-se que os produtores com propriedades maiores possuem mais áreas contratadas, visando maiores retornos financeiros investindo na expansão da produção de madeira.

A inferência anterior é subsidiada pelos argumentos de que os produtores com maior extensão de terras podem alocar mais hectares para a prática de fomento florestal, considerando-se que poderá utilizar o restante de sua propriedade na produção de outras culturas.

Rochadelli et al.(2008) afirmaram, no entanto, que, a partir de determinado tamanho da propriedade e do nível de desenvolvimento tecnológico dos produtores rurais, estes passaram a atuar de forma independente na produção de madeira, deixando a prática de fomento. Desse modo, reforça-se a maior necessidade de elaboração de políticas especificas para o desenvolvimento do fomento florestal e também dos produtores rurais, que estão diretamente vinculados a ele.

Além dos coeficientes para explicar o poder de classificação da função discriminante, pode-se utilizar a correlação entre a função e as variáveis explicativas para identificar aquelas que melhor discriminam os grupos.

Na Tabela 3, apresentam-se, desse modo, os coeficientes de correlação das variáveis com a função, em que se constatou que os fatores mais importantes para classificar os produtores nos grupos são, respectivamente, $\operatorname{SISMED}(0,681), \operatorname{ACONTRAT}(0,679)$ e TAMPROP $(0,461)$.

A função discriminante viabiliza a construção de escores (pontuação) para cada produtor. Para o cálculo do escore, basta substituir os valores das respostas na função e identificar aqueles que se apresentam mais próximos da troca de grupos, ou seja, aqueles mais propensos a saírem de um grupo e juntarem a outro. O ponto de corte, ou $\mathrm{Z}$ crítico calculado, foi de -0,00036. Assim, os objetos que apresentaram escores superiores a esse valor pertencem ao grupo 1, e valores inferiores são classificados no grupo 2 .
Na Tabela 4, apresenta-se a adequabilidade do modelo quanto à classificação dos produtores nos grupos definidos. Desse modo, 70,2\% dos produtores foram classificados corretamente em suas populações, percentual esse considerado satisfatório para esse tipo de estudo.

Tabela 3 - Correlação entre a função discriminante (Y) e as variáveis explicativas TAMPROP, ACONTRAT e SISMED

Table 3 - Correlation between discriminating function $(Y)$ and the explanatory variables TAMPROP, ACONTRAT and SISMED

\begin{tabular}{lc}
\hline Variáveis & Correlação \\
\hline TAMPROP - Tamanho da propriedade & 0,681 \\
ACONTRAT - Área contratada & 0,679 \\
SISMED - Sistema de medição & 0,461 \\
\hline
\end{tabular}

Fonte: Dados da pesquisa.

Tabela 4-Teste de adequabilidade do modelo quanto à classificação dos produtores nos grupos

Table 4-Model adequability test for producer classification by group

\begin{tabular}{|c|c|c|c|c|c|}
\hline \multirow{2}{*}{\multicolumn{2}{|c|}{ Grupo }} & & \multicolumn{2}{|c|}{ Previsto } & \multirow[t]{2}{*}{ Total* } \\
\hline & & & Novos & Apenas um & \\
\hline \multirow[t]{4}{*}{$\overline{\text { Original }}$} & Unidades & $\begin{array}{l}\text { Novos } \\
\text { contratos }\end{array}$ & 55 & 25 & 80 \\
\hline & & $\begin{array}{l}\text { Apenas um } \\
\text { contrato }\end{array}$ & 11 & 30 & 41 \\
\hline & $(\%)$ & $\begin{array}{l}\text { Novos } \\
\text { contratos }\end{array}$ & 68,8 & 31,2 & 100 \\
\hline & & $\begin{array}{l}\text { Apenas um } \\
\text { contrato }\end{array}$ & 26,8 & 73,2 & 100 \\
\hline
\end{tabular}

Fonte: Dados da pesquisa.

$* 70,2 \%$ dos produtores foram corretamente classificados.

\section{CONCLUSÕES}

O modelo estimado é satisfatório para realizar a discriminação dos produtores entre os grupos prédefinidos. Por meio dele, verificou-se que os sistemas de medição da madeira (duvidosos na visão dos produtores) influenciam a decisão de realizar novos contratos. Desse modo, sugere-se que as empresas que praticam o fomento florestal desenvolvam mecanismos de acompanhamento técnico e de formação dos produtores, no intuito de aumentar a confiança deles com a empresa. Assim, com maior conhecimento do processo de corte e medição, os produtores reduzirão as expectativas negativas, aumentando as chances favoráveis de realização de novos contratos. 
As outras variáveis que apresentaram significância estatística no modelo se referem à área contratada e ao tamanho da propriedade. A partir dos coeficientes da função, conclui-se que, quanto maior a área contratada, maiores as chances de realização de novos contratos. E, por fim, os produtores donos de propriedades maiores possuem mais chances de realizar contratos, devido à disponibilidade de áreas para o plantio e também aos benefícios provenientes da atividade, como maior retorno financeiro e disponibilidade de recursos (materiais e financeiros) para investimento no plantio de novas florestas.

As variáveis citadas apresentaram correlações positivas dos indivíduos dentro dos seus grupos. Assim, confirma-se o poder de discriminação da função estimada.

\section{AGRADECIMENTO}

\section{Ao CNPq e a FAPEMIG.}

\section{REFERENCIAS}

ASSOCIACAO DE PRODUTORES DE FLORESTAS PLANTADAS - ABRAF. Anuário estatístico 2006: Ano base 2005. Disponível em: $<\underline{\text { http:// }}$ www.abraflor.org.br/estatisticas/anuario-ABRAF2006.pdf>. Acesso em: 29 maio 2007.

\section{BARROSO. L. P.; ARTES, R. Análise}

multivariada. Lavras, Universidade Federal de Lavras, 2003. 156p. (Não Publicado)

CANTO, J. L. et al. Colheita e transporte florestal em propriedades rurais fomentadas no estado do Espírito Santo. Revista Árvore, v.30, n.6. p.989-998, 2006.

COELHO, A. B.; FERNANDES, E. A.; SAMPAIO, D. O. Agronegócio e investimento direto estrangeiro: Uma análise do impacto sobre as exportações brasileiras.. In: CONGRESSO DA SOCIEDADE BRASILEIRA DE ECONOMIA, ADMINISTRAÇÃO E SOCIOLOGIA RURAL, 2008, Rio Branco. Anais... Rio Branco: 2008.

GIL, A. C. Métodos e técnicas de pesquisa social. 5.ed. São Paulo: Atlas, 1999. 208p.

GIMENES, R. M. T.; URIPE-OPAZO, M. A. Modelos multivariantes para a previsão de insolvência em cooperativas agropecuárias: Uma comparação entre a análise discriminante e a análise de probabilidade condicional - Logit. Caderno de Pesquisas em Administração, v.8, n.3. jul/set, 2001.
GONÇALVES, C. A.; DIAS, A. T.; MUNIZ, R. M. Análise discriminantes das relações entre fatores estratégicos, indústria e desempenho em organizações brasileiras atuantes na indústria manufatureira. Revista de Administração Contemporânea, v. 12, n.2, p.287-311, 2008.

HAIR, J. F.et al. Análise multivariada de dados. 5.ed. Porto Alegre: Bookman, 2005.

MARoco, J. Análise estatística: com a utilização do SPSS. Lisboa: Sílabo, 2003.

Mingote, S.A. Análise de dados através de métodos de estatísticas

multivariada: uma abordagem aplicada. Belo Horizonte: Universidade Federal de Minas Gerais, 2005. 297p.

NANNI, M. R.; DEMATLÊ, J. A.; FIORIO, P. R. Análise discriminante dos solos por meio da resposta espectral no nível terrestre. Pesquisa Agropecuária Brasileira, v.39, n.10, p.995-1006, 2004.

OLIVEIRA, P. R. S. Diagnóstico e indicadores de sustentabilidade em fomento florestal no estado do Espírito do Santo. 2003. 127f. Dissertação (Mestrado em Ciência Florestal) - Universidade Federal de Viçosa, Viçosa, MG, 2003.

OLIVEIRA, P. R. S.; VALVERDE, S. R.; COELHO, F. M. G. Aspectos de relevância econômica no fomento florestal a partir da recepção dos produtores rurais envolvidos. Revista Árvore, v.30, n.4, p.593-601, 2006.

PINARE, A. G. V. Uso da função

discriminante linear na

classificação dos fatores que determinam o êxodo rural. Petrolina: Embrapa-CPATSA/SUDENE, 1985. 30p.

SILVA, F. L. Estudo da relação de confiança em programa de fomento florestal de indústria de celulose na visão dos produtores rurais. 2007. 102f. Dissertação (Mestrado em Ciência Florestal) - Universidade Federal de Viçosa, Viçosa, MG, 2007.

R. Árvore, Viçosa-MG, v.33, n.2, p.377-386, 2009 
SILVA, E. Avaliação qualitativa de impactos ambientais do reflorestamento no Brasil. 1994. 309f. Tese (Doutorado em Ciência Florestal) Universidade Federal de Viçosa, Viçosa, MG, 1994.

SOCIEDADEBRASILEIRADESILVICULTURA-SBS. Fatos e números do Brasil Florestal.

Disponível em: <http://www.sbs.org.br/ FatoseNumeros doBrasilFlorestal.pdf $>$. Acesso em: 28 maio. 2007.
ROCHADELLI, R. et al. Expansão florestal via fomento no segundo planalto paranaense: Uma abordagem a partir da estrutura fundiária das propriedades rurais da região. Cerne, v.14, n.2, p.163-169, 2008.

VALVERDE, S. R. et al. Efeitos multiplicadores da economia florestal brasileira. Revista Árvore, v.27, n.3, p.285-293, 2003.

R. Árvore, Viçosa-MG, v.33, n.2, p.377-386, 2009 